\title{
On coherent sets and the transmission of confirmation
}

\author{
Franz Dietrich and Luca Moretti \\ September $2004^{1}$
}

\begin{abstract}
In this paper, we identify a new and mathematically well-defined sense in which the coherence of a set of hypotheses can be truth-conducive. Our focus is not, as usually, on the probability but on the confirmation of a coherent set and its members. We show that, if evidence confirms a hypothesis, confirmation is "transmitted" to any hypotheses that are sufficiently coherent with the former hypothesis, according to some appropriate probabilistic coherence measure such as Olsson's or Fitelson's measure. Our findings have implications for scientific methodology, as they provide a formal rationale for the method of indirect confirmation and the method of confirming theories by confirming their parts.
\end{abstract}

\section{Introduction}

Many epistemologists find it intuitive that coherence is truth-conducive. However, it has not yet been possible to turn this plausible intuition into an exact claim without facing serious objections or counterexamples. For instance, if the truth-conduciveness of coherence is understood as the claim that more coherent sets of statements (propositions, beliefs, scientific hypotheses, etc.) are always more likely to be true than less coherent ones, the thesis is obviously false. For, "a well-composed novel is usually not true, and yet it may still be highly coherent - perhaps far more so than reality in itself." (Olsson 2002, 247). The problem of truth-conduciveness of coherence has been investigated in a long, open and on-going debate, by, among others, BonJour 1985, Klein and Warfield 1994 and 1996, Merricks 1995, Cross 1999, Shogenji 1999 and 2001, Akiba 2000, Olsson 2001 and 2002, Bovens and Hartmann 2003 and 2004. Since the thesis "more coherent sets have higher probability" is not true as such, the general strategy in the literature has been to argue that the thesis becomes true once restricted to sets satisfying suitable conditions. However, the nature and even existence of such conditions is highly controversial.

This paper takes a different approach, by focusing not on the probability but on the confirmation of coherent sets. This will lead to a sense in which coherence is truth-conducive, a sense that is less ambitious than the claim "coherence increases probability" but is mathematically well-defined. ${ }^{2}$ We define a property, to be called

\footnotetext{
${ }^{1}$ We wish to thank Claus Beisbart, Ludwig Fahrbach, Branden Fitelson, Stephan Hartmann and Franz Huber for very inspiring discussions. We are also grateful to the Alexander von Humboldt Foundation, the Federal Ministry of Education and Research, and the Program for the Investment in the Future (ZIP) of the German Government, for supporting this research.

${ }^{2}$ We should mention that Bovens and Hartmann 2002 also identify a mathematically well-defined, yet somewhat different and special sense of truth-conduciveness of coherence. They focus on information sets, specifically on sets of hypotheses that have been confirmed by independent and equally
} 
confirmation transmission, to the effect that, in short, if some evidence confirms a given member of a sufficiently coherent set of hypotheses, then it also confirms each other member. Our truth-conduciveness is thus a conditional one: coherence is truth-conducive conditional on evidence confirming a member of the set. Our findings establish a link between the Bayesian theories of confirmation and of coherence (for Bayesian confirmation theory, see for instance Fitelson 2000, 2001).

Specifically, we define confirmation transmission as a property of the degree of coherence of sets as given by some probabilistic coherence measure (as opposed to some absolute, i.e. ungraded, notion of coherence). Among the different coherence measures recently proposed in the literature, some but not all satisfy confirmation transmission. In particular, we prove that Olsson's measure satisfies a strong form of confirmation transmission, Fitelson's measure satisfies a weaker form of confirmation transmission, and Shogenji's measure violates even the weaker property. We show that if a coherence measure satisfies different confirmation transmission properties, then coherence becomes in well-defined ways truth-conducive and relevant for scientific methodology. But we do not argue that our confirmation transmission properties are in all circumstances essential requirements on a coherence measure, since a measure may be used for a different purpose than confirmation transmission.

In Section 2, we introduce the formal framework and the notion of a coherence measure; and we define the coherence measures referred to in this paper. In Section 3 , we show that the coherence of a set of hypotheses (e.g. a theory) can be used to ascertain the confirmation of the set by evidence: if some element of a set is confirmed and the set is sufficiently coherent, then confirmation is 'transmitted' to the other members of the set, and to their conjunction. In Section 4, we show that confirmation transmission can be used to justify the method of indirect confirmation. Section 5 contains the conclusions of the paper.

\section{Probabilistic measures of coherence}

Intuitively, a set of statements is coherent if its members 'hang together' (C. I. Lewis 1946). Moreover, coherence is generally thought to be a matter of degrees. Recently, a number of probabilistic coherence measures have been proposed, which are supposed to account for both of these intuitions. A coherence measure is a function that assigns to each (finite non-empty) set of statements a real number, interpreted as the (degree of) coherence of that set. The proposed measures contrast with each other in that they induce significantly different coherence orderings.

More precisely, consider a standard formal language of propositional logic (with at least the connectives $\neg, \wedge, \vee$ ). Coherence measures are defined relative to a finitely additive Kolmogorov probability function $P$ on the language (usually interpreted as reflecting the subjective probabilities of a real or idealised agent). For simplicity, we assume that $P$ is regular ${ }^{3}$, and we restrict attention to the coherence of sets of

(partially) reliable sources. They define a sophisticated partial cohernce ordering over information sets, and show that one information set is at least as probable as another if it is at least as coherent as the other and in addition (i) both sets have equal size, (ii) the different pieces of information come from independent and equally reliable sources, and (iii) both sets had the same probability prior to being reported by the sources (cf. p. 626).

${ }^{3}$ I.e., $P(H)>0$ for every consistent formula $H$. Our results do not depend on this assumption, 
consistent formulae. Specifically, a coherence measure is a function $C$ that maps every non-empty finite set $S$ of individually consistent formulae to a number $C(S) \in \mathbf{R}$. Thus $C: \mathcal{S} \rightarrow \mathbf{R}$, where $\mathcal{S}$ is the set of all non-empty finite sets $S$ of consistent formulae.

To exemplify our confirmation transmission properties, we focus on the coherence measures proposed by, respectively, Shogenji 1999, Olsson 2002 and Fitelson 2004.

- Shogenji defines the coherence of a set $S \in \mathcal{S}$ as

$$
C_{\mathrm{S}}(S):=\frac{P\left(\wedge_{H \in S} H\right)}{\Pi_{H \in S} P(H)} .
$$

This measure ranges over the interval $[0, \infty)$. If $S$ is inconsistent then $C_{\mathrm{S}}(S)=0$ (minimal coherence). If $S$ consists of probabilistically independent formulae, $C_{\mathrm{S}}(S)=$ 1. Shogenji interprets $S$ as simply "coherent" or "incoherent" if $C_{\mathrm{S}}(S)>1$ or $C_{\mathrm{S}}(S)<$ 1 , respectively.

- Olsson defines the coherence of a set $S \in \mathcal{S}$ as

$$
C_{\mathrm{O}}(S):=\frac{P\left(\wedge_{H \in S} H\right)}{P\left(\vee_{H \in S} H\right)} .
$$

This measure ranges over the interval $[0,1]$. If $S$ is inconsistent then $C_{\mathrm{O}}(S)=0$ (minimal coherence), and if $S$ consists of pairwise logically equivalent formulae then $C_{\mathrm{O}}(S)=1$ (maximal coherence).

- Fitelson defines the coherence of a set $S \in \mathcal{S}$ as the average degree to which conjunctions of non-empty subsets $S_{1} \subseteq S$ are supported by conjunctions of other non-empty subsets $S_{2} \subseteq S \backslash S_{1}$. Specifically, he defines ${ }^{4}$

$$
C_{\mathrm{F}}(S):=\frac{1}{|\mathcal{R}|} \sum_{\left(S_{1}, S_{2}\right) \in \mathcal{R}} F\left(\wedge_{H_{1} \in S_{1}} H_{1}, \wedge_{H_{2} \in S_{2}} H_{2}\right),
$$

where $\mathcal{R}$ is the set of all pairs $\left(S_{1}, S_{2}\right)$ of non-empty subsets $S_{1}, S_{2} \subseteq S$ with $S_{1} \cap S_{2}=\emptyset$ (there are exactly $|\mathcal{R}|=n\left(2^{n-1}-1\right)$ such pairs), and $F$ is Kemeny and Oppenheim's 1952 measure of factual support; specifically

$$
F(H, K):=\frac{P(K \mid H)-P(K \mid \neg H)}{P(K \mid H)+P(K \mid \neg H)},
$$

interpreted as -1 if $K \vDash \neg H$, and as 1 if $K \vDash H$ and $K$ is not a contradiction. ${ }^{5}$ For instance, if $S=\left\{H, H^{*}\right\}\left(H \neq H^{*}\right)$ then $\mathcal{R}=\left\{\left(\{H\},\left\{H^{*}\right\}\right),\left(\left\{H^{*}\right\},\{H\}\right)\right\}$, and so

$$
C_{\mathrm{F}}(S)=\frac{1}{2}\left[F\left(H, H^{*}\right)+F\left(H^{*}, H\right)\right] .
$$

Fitelson's measure ranges over the interval $[-1,1]$. If $S$ consists of pairwise inconsistent formulae then $C_{\mathrm{F}}(S)=-1$ (minimal coherence). If $S$ consists of pairwise equivalent formulae then $C_{\mathrm{F}}(S)=1$ (maximal coherence). If $S$ consists of probabilistically independent formulae then $C_{\mathrm{F}}(S)=0$.

which one may want to drop to allow for propositional background knowledge.

${ }^{4}$ If $|S|=1$ we have $\mathcal{R}=\emptyset$, so that expression in (1) is undefined. In this case we suggest defining $C_{\mathrm{F}}(\{H\}):=1$, in accordance with Fitelson's aim that sets of pairwise equivalent (consistent) formulae should have maximal coherence.

${ }^{5}$ We use Fitelson's revised definition of his coherence measure, presented by him at Bayesian Epistemology conference (London School of Economics and Political Science, UK, 28 June 2004). 


\section{Confirming theories by confirming their parts}

It is well-know that the confirmation of individual members of a set of statements does not entail confirmation of the entire set, i.e. of the conjunction of its members. In particular, if a theory is interpreted as a set of (scientific) hypotheses, ${ }^{6}$ and the different hypotheses are individually confirmed, it does not in general follow that the theory as a whole is confirmed. Here, a formula $E$ is said to (incrementally) confirm a formula $H$ if $P(E)>0$ and $P(H \mid E)>P(H)$.

It is even easy to construct cases, within or outside science, in which evidence confirms each part of a theory yet disconfirms the theory. We give two examples.

First, assume Anne has two lovers, Peter and Sam, who don't know of each other. Yesterday there was a party, and suppose that a theory says that Peter and Sam both were at this party. Now, we learn that Anne was at the party. This is evidence for both parts of the theory: it increases the probability that Peter was at the party and increases the probability that Sam was at the party. But it decreases the probability of the entire theory, because Anne would never have gone to a party where both of her lovers are present.

Second, consider a physical experiment that involves two sources. Most likely, none of the sources emits a particle; but each source may, with a small probability, emit either an electron or a positron. A physicist is interested in whether it is true that both sources emit an electron; so, she wants to know whether the first source emits an electron $(H)$ and the second source emits an electron $\left(H^{*}\right)$. She observes two photons $(E)$ indicating an annihilation. Annihilation is possible only if the two sources do emit particles, but particles of opposite charges (one emits an electron, the other a positron). Hence the observation $E$ confirms $H$ and confirms $H^{*}$, yet disconfirms $H \wedge H^{*}$.

In general, are there conditions on a set $S$ under which it is justified to consider $S$ as confirmed by an evidence $E$ that confirms a member of $S$ ? We show that sufficiently high coherence of $S$ is such a condition (not a necessary condition, of course). But "sufficiently high" with respect to which coherence measure $C$ ? There are many plausible ways to measure coherence, but not for all of them the claim "sufficiently high coherence transmits confirmation" holds. Below, we prove, as an example, that the claim does hold for a particularly elementary coherence measure: Olsson's measure (defined above).

Let us now define two properties, satisfied by some but not all coherence measures $C$.

Confirmation Transmission (CT). For any formulae $E, H$ such that $E$ confirms $H$, there exists a (non-trivial ${ }^{7}$ ) coherence threshold $c=c_{E, H} \in \mathbf{R}$ such that, for any set $S \in \mathcal{S}$ for which $H \in S$ and $C(S) \geq c, E$ confirms each member of $S$.

Confirmation Transmission to the Conjunction (CTC) For any formulae

\footnotetext{
${ }^{6}$ Sometimes, a theory is required to be closed under logical entailment (hence in particular infinite). Our notion of a theory is that of a set of hypotheses or axioms (the deductive closer of which is a theory in the above sense).

${ }^{7}$ By "non-trivial" we mean that $c<\sup _{S \in \mathcal{S} \& S \ni H} C(S)$. This supremum equals $\sup _{S \in \mathcal{S}} C(S)$ (the maximal coherence level) if $C$ is $C_{\mathrm{S}}$ or $C_{\mathrm{O}}$ or $C_{\mathrm{F}}$ (and, generally, if $C$ assigns maximal coherence to any set of pairwise equivalent formulae).
} 
$E, H$ such that $E$ confirms $H$, there exists a (non-trivial ${ }^{7}$ ) coherence threshold $c=$ $c_{E, H} \in \mathbf{R}$ such that, for any set $S \in \mathcal{S}$ for which $H \in S$ and $C(S) \geq c, E$ confirms the conjunction $\wedge_{H^{*} \in S} H^{*}$.

In this section, (CTC) is the main focus. However, a useful step towards proving that a coherence measure satisfies (CTC) is to show first that it satisfies (CT). The reason is given by the following result:

Theorem 1 Every coherence measure $C$ that satisfies $(C T)$ and $C\left(S \cup\left\{\wedge_{H \in S} H\right\}\right) \geq$ $C(S)$ for every $S \in \mathcal{S}$ also satisfies (CTC) (and each possible coherence threshold $c_{E, H}$ in $(C T)$ is a possible coherence threshold $c_{E, H}$ in $\left.(C T C)\right)$.

Proof. Assume $C$ satisfies (CT) and the inequalities $C\left(S \cup\left\{\wedge_{H \in S} H\right\}\right) \geq C(S)$ for every $S \in \mathcal{S}$. Let $E$ confirm $H$, and let $c_{E, H}$ be as given in $(\mathrm{CT})$. To show (CTC), consider any set $S \in \mathcal{S}$ such that $H \in S$ and $C(S) \geq c_{E, H}$. By assumption, $C\left(S \cup\left\{\wedge_{H \in S} H\right\}\right) \geq C(C)$, and so $C\left(S \cup\left\{\wedge_{H \in S} H\right\}\right) \geq c_{E, H}$. Hence, by (CT), $E$ confirms each member of $S \cup\left\{\wedge_{H \in S} H\right\}$, in particular $\wedge_{H \in S} H$. This proves (CTC).

We now apply Theorem 1 to Olsson's coherence measure $C_{\mathrm{O}}$. As shown in the appendix, $C_{\mathrm{O}}$ satisfies (CT). As $C_{\mathrm{O}}$ also satisfies the inequalities in Theorem 1 (as $C_{\mathrm{O}}(S)=C_{\mathrm{O}}\left(S \cup\left\{\wedge_{H \in S} H\right\}\right)$ for each $\left.S \in \mathcal{S}\right)$, it follows that $C_{\mathrm{O}}$ satisfies (CTC):

Theorem 2 Olsson's coherence measure $C_{O}$ satisfies $(C T)$ and $(C T C)$, with coherence threshold in both cases given by $c_{E, H}=\frac{1}{1+P(E \mid H)-P(E)}$.

Note that the coherence threshold $c=\frac{1}{1+P(E \mid H)-P(E)}$ is the higher, the less dependent $E$ and $H$ are in the sense that $P(E \mid H)-P(E)$ is smaller. As expected, when $P(E \mid H)-P(E)$ tends to 0 (independence), $c$ tends to 1 (maximal coherence).

To illustrate the importance of (CTC) for the confirmation of sets, suppose that $S \in \mathcal{S}$ is the set of hypotheses of a theory (or of points of the charge in a law suit). Each $H \in S$ has been confirmed by some experiments (or witness reports). Should one consider the whole theory (or charge) $\wedge_{H^{*} \in S} H^{*}$ as confirmed by each experiment (or witness report)? This depends on how coherent the theory (or charge) is, as measured by some coherence measure $C$ satisfying $\left(\mathrm{CT}^{*}\right)$ (for instance, Olsson's measure). More precisely, each experiment (or witness report) $E$ confirming a hypothesis $H$ such that $C(S) \geq c_{E, H}$ also confirms the entire theory (or charge) $\wedge_{H^{*} \in S} H^{*}$; whereas each experiment (witness report) $E$ confirming a hypothesis $H$ such that $C(S)<c_{E, H}$ may or may not confirm the entire theory (or charge) $\wedge_{H^{*} \in S} H^{*}$.

In should be emphasised, however, that a high enough coherence of a set $S$ is sufficient but not necessary for transmission of confirmation from a member of $S$ to the conjunction of $S$.

\section{A rationale for the method of indirect confirmation}

While in the last section we focussed on the confirmation of sets of hypotheses, we now turn to the confirmation a single (scientific) hypothesis via indirect confirmation. 
This is a second sense in which confirmation transmission is relevant to scientific methodology.

It is common scientific practice to consider a hypothesis $H^{*}$ as confirmed by evidence $E$ if $E$ confirms some other hypothesis $H$ related to $H^{*}$. This is called the method of indirect confirmation (e.g. Laudan and Leplin 1991, Hoefer and Rosenberg 1994). The method is used in cases where it is not immediately obvious that $E$ confirms $H^{*}$, but it is clear that $E$ confirms $H$ (for instance because $E$ is a logical consequence of $H$, possibly together with auxiliaries). As explained below, the method of indirect confirmation was for example used to argue that magnetic striping on ocean floors confirms the climate change hypothesis. The method of indirect confirmation was also proposed as a way to decide between empirically equivalent theories. Laudan and Leplin 1991 and Hoefer and Rosenberg 1994 argue that two empirically equivalent theories need not be empirically underdetermined, as one of them may have more indirect support than the other.

But what exactly does it mean that $H$ and $H^{*}$ are "related"? Defining "related" in logical terms (either by $H \vDash H^{*}$ or by $H^{*} \vDash H$ ) is inappropriate, since $E$ can confirm $H$ without confirming $H^{*} .8$ Let us therefore interpret "related" as "coherent". This allows us to provide a formal justification of the method of indirect confirmation: if $E$ confirms $H$ and $C$ is some coherence measure satisfying confirmation transmission (CT) for which $C\left(\left\{H, H^{*}\right\}\right)$ is sufficiently high (specifically, at least $c_{E, H}$ ), then $E$ confirms $H^{*}$.

Note that this argument appeals to $(\mathrm{CT})$ only in the special case of the binary set $S=\left\{H, H^{*}\right\} .{ }^{9}$ So, the argument remains true even if $C$ does not satisfy (CT) but only the following less demanding notion of confirmation transmission:

Weak Confirmation Transmission $\left(\mathbf{C T}^{*}\right)$. For any formulae $E, H$ such that $E$ confirms $H$, there exists a (non-trivial ${ }^{10}$ ) coherence threshold $c=c_{E, H} \in \mathbf{R}$ such that, for any consistent formula $H^{*}$ for which $C\left(\left\{H, H^{*}\right\}\right) \geq c, E$ confirms $H^{*}$.

As our formal account of the method of indirect confirmation requires not (CT) but only $\left(\mathrm{CT}^{*}\right)$, it is open to more coherence measures, including Fitelson's and Olsson's ones: ${ }^{11}$

\footnotetext{
${ }^{8}$ The logical interpretation of "related" given by Laudan and Leplin 1991 leads into the Hempelian paradox of confirmation that everything confirms everything, as shown by Okasha 1997.

${ }^{9} \mathrm{~A}$ formal account of the following more general method of indirect confirmation would, however, require $C$ to satisfy $(\mathrm{CT})$ (not just $\left(\mathrm{CT}^{*}\right)$ ): if $E$ confirms $H$, and $H$ and $H^{*}$ both belong to some set $S$ of hypotheses with coherence $C(S) \geq c_{E, H}$, then $E$ confirms $H^{*}$.

${ }^{10}$ By "non-trivial" we mean that $c<\sup _{H^{*} \text { consistent }} C\left(\left\{H, H^{*}\right\}\right)$. This supremum equals $\sup _{S \in \mathcal{S}} C(S)$ (the maximal coherence level) if $C$ is $C_{\mathrm{O}}$ or $C_{\mathrm{F}}$ (or, more generally, if $C$ assigns maximal coherence to any set of pairwise equivalent formulae), but equals $\frac{1}{P(H)}$ if $C$ is $C_{\mathrm{S}}$ (see the proof of Theorem 4).

${ }^{11}$ To see why $C_{\mathrm{F}}$ violates $(\mathrm{CT})$ (if the language contains at least two atomic formulae), let $E$ confirm $H$ but not confirm $H^{*}$, where $H \wedge H^{*}$ is consistent (such $E, H, H^{*}$ exist by assumption on the language). Suppose for contradiction that there is a (non-trivial) threshold $c_{E, H}$ such that, for all sets $S \in \mathcal{S}$, if $H \in S$ and $C(S) \geq c_{E, H}$ then $E$ confirms each member of $S$. Let $H_{1}, H_{1}^{*}, H_{2}, H_{2}^{*}, H_{3}, H_{3}^{*}, \ldots$ be distinct formulae such that each $H_{i}$ is equivalent to $H$ and each $H_{i}^{*}$ is equivalent to $H^{*}$. For each $n \in\{1,2, \ldots\}$ consider the set $S_{n}:=\left\{H, H^{*}, H_{1}, H_{1}^{*}, \ldots, H_{n}, H_{n}^{*}\right\}$. By definition of $C_{\mathrm{F}}, C_{\mathrm{F}}\left(S_{n}\right)$ is the average of all terms of the form $F\left(\wedge_{K_{1} \in S_{1}} K_{1}, \wedge_{K_{2} \in S_{2}} K_{2}\right)$, where $\left(S_{1}, S_{2}\right)$ ranges over $\mathcal{M}_{n}$, the set of pairs of disjoint non-empty subsets of $S_{n}$. Note that $\wedge_{K_{1} \in S_{1}} K_{1}$ is (depending on $S_{1}$ ) equivalent either to $H$, or to $H^{*}$, or to $H \wedge H^{*}$; similarly, $\wedge_{K_{2} \in S_{2}} K_{2}$ is equivalent either to $H$, or to $H^{*}$, or to $H \wedge H^{*}$.
} 
Theorem 3 Fitelson's and Olsson's coherence measures both satisfy (CT*), with coherence threshold given by, respectively, $c_{E, H}=\frac{1}{1+P(E \wedge H)-P(E) P(H)}$ and $c_{E, H}=$ $\frac{1}{1+P(E \mid H)-P(E)}$.

(The proof is in the appendix.) Our earlier comments on the threshold for Olsson's measure apply similarly to the threshold of Fitelson's measure: it is the larger, the nearer $E$ and $H$ are to being independent (in the sense that $P(E \wedge H)-P(E) P(H)$ is smaller), and it tends to 1 (maximal coherence) as $P(E \wedge H)-P(E) P(H)$ tends to 0 (full independence).

Shogenji's coherence measure $C_{\mathrm{S}}$, however, does not satisfy $\left(\mathrm{CT}^{*}\right)$, and hence $C_{\mathrm{S}}$ cannot be used to formalise the method of indirect confirmation.

Theorem 4 Shogenji's coherence measure $C_{S}$ does not satisfy $\left(C T^{*}\right)$ (provided that the language has at least two distinct atomic formulae).

(The proof is in the appendix.)

Let us give a historical example of indirect confirmation. The so-called continental drift theory $(H)$ states, roughly, that the earth's surface is composed by a number of oceanic and continental plates that move in time as they float on top of the asthenosphere. The theory was not accepted until the 1960s, when it was strongly confirmed by the systematic observation of magnetic striping on ocean floors $(E)$. Briefly, on both sides of mid-ocean ridges, wide stripes of magmatic rock with alternating polarity were observed. It was already established by then that earth's magnetic polarity reversed at certain geologic times and that, as new magma wells up out of a rift, it gets magnetised in the direction of earth's polarity at that time. So, magnetic striping was interpreted as providing a record of a spreading movement of the ocean floor over time: a confirmation of the continental drift theory.

The theory of continental drift is coherent with the climate change hypothesis $\left(H^{*}\right)$ whereby the climate of continents has varied throughout geologic time. $H^{*}$ was a plausible but little confirmed hypothesis before the 1960s. The discovery of magnetic striping was taken to confirm the climate change hypothesis. As argued in Laudan and Leplin 1991, this was a case of indirect confirmation: $E$ confirms $H^{*}$ as $H^{*}$ is closely related with $H$ which is confirmed by $E .^{12}$ A formal explanation that $E$ indeed confirms $H^{*}$ would be that $E$ confirms $H$ and $C\left(\left\{H, H^{*}\right\}\right) \geq c_{E, H}$ for some coherence measure $C$ satisfying $\left(\mathrm{CT}^{*}\right)$ (for instance, Olsson's or Fitelson's but not Shogenji's measure).

One easily verifies that, as $n$ increases, the proportion of pairs $\left(S_{1}, S_{2}\right)$ in $\mathcal{M}_{n}$ for which $\wedge_{K_{1} \in S_{1}} K_{1}$ and $\wedge_{K_{2} \in S_{2}} K_{2}$ are each equivalent to $H \wedge H^{*}$ tends to 1 . So the proportion of pairs $\left(S_{1}, S_{2}\right)$ in $\mathcal{M}_{n}$ for which $F\left(\wedge_{K_{1} \in S_{1}} K_{1}, \wedge_{K_{2} \in S_{2}} K_{2}\right)=1$ tends to 1 , and hence $C_{\mathrm{F}}\left(S_{n}\right) \rightarrow 1$. Therefore, for sufficiently large $n, C_{\mathrm{F}}\left(S_{n}\right) \geq c_{E, H}$, however close to $1 c_{E, H}$ is. Yet $E$ does not confirm $H^{*} \in S_{n}$. However, we conjecture that $F_{\mathrm{O}}$ satisfies the following condition, which is stronger than $\left(\mathrm{CT}^{*}\right)$ but weaker than (CT): for any fixed $n \in\{2,3, \ldots\}$ and any formulae $E, H$ such that $E$ confirms $H$, there exists a (non-trivial) coherence threshold $c=c_{E, H} \in \mathbf{R}$ such that, for any set $S \in \mathcal{S}$ of size $n$ for which $H \in S$ and $C(S) \geq c, E$ confirms each member of $S$.

${ }^{12}$ In fact, Laudan and Leplin provide a slightly different reconstruction of case. 


\section{Conclusion}

The literature about coherence is far from an agreement on how to measure the coherence of a set (of statements, scientific hypotheses etc.). One of the reasons is that role of coherence is controversial. Despite attempts to argue that the coherence of a set can, under suitable conditions, imply that the set is probable, none of the coherence measures in the literature reflects this claim in any straightforward and general way. In this paper, we have shown that, in the different context of the confirmation of hypotheses, coherence can be given a simple and mathematically welldefined significance, which is reflected in some of the coherence measures proposed so far, and probably in many other ones that have yet to be devised. Specifically, we show that Olsson's measure satisfies (CT) and (CTC), and Fitelson's measure satisfies $\left(\mathrm{CT}^{*}\right)$, whereas Shogenji's measure violates all these conditions. Satisfaction or violation of our conditions is not a reason to accept or reject a coherence measure in general. Indeed, if the purpose is not confirmation transmission, one may prefer Shogenji's and Fitelson's measures over Olsson's measure on the grounds that they always assign a higher coherence to a positively dependent set than to an independent set (see Fitelson 2003).

The relevance of our confirmation transmission properties for scientific methodology is that they provide formal rationales for two standard procedures: the method of confirming a theory by confirming its parts, and the method of indirect confirmation. Indeed, if coherence is defined in accordance with (CTC), evidence confirming a part of a sufficiently coherent theory also confirms the theory as a whole. Moreover, if coherence is defined in accordance with $\left(\mathrm{CT}^{*}\right)$, evidence confirming a hypothesis sufficiently coherent with another hypothesis also confirms the latter hypothesis.

\section{Appendix: proof of the theorems}

Proof of Theorem 2. We need only show the claim relating to (CT), as this claim implies that relating to (CTC) by using Theorem 1.

Let $E, H$ be formulae such that $E$ confirms $H$, i.e. $P(H \mid E)-P(H)>0$, and put $c:=\frac{1}{1+P(E \mid H)-P(E)}$. Consider any set $S \in \mathcal{S}$ such that $H \in S$ and $C_{\mathrm{O}}(S) \geq c$. Take any $H^{*} \in S \backslash\{H\}$, and let us show that $E$ confirms $H^{*}$, i.e. that $D^{*}:=P\left(H^{*} \mid E\right)-$ $P\left(H^{*}\right)>0$.

1. By the definition of $C_{\mathrm{O}}, C_{\mathrm{O}}\left(\left\{H, H^{*}\right\}\right) \geq C_{\mathrm{O}}(S)$. So $C_{\mathrm{O}}\left(\left\{H, H^{*}\right\}\right) \geq c$, or

$$
\frac{1}{C_{\mathrm{O}}\left(\left\{H, H^{*}\right\}\right)} \leq \frac{1}{c}=1+P(E \mid H)-P(E) .
$$

Consider the formula $K$ defined as $\left(H \wedge \neg H^{*}\right) \vee\left(\neg H \wedge H^{*}\right)$. We have

$$
\begin{aligned}
\frac{1}{C_{\mathrm{O}}\left(\left\{H, H^{*}\right\}\right)} & =\frac{P\left(H \vee H^{*}\right)}{P\left(H \wedge H^{*}\right)}=\frac{P\left(H \wedge H^{*}\right)+P(K)}{P\left(H \wedge H^{*}\right)} \\
& =1+\frac{P(K)}{P\left(H \wedge H^{*}\right)} \geq 1+\frac{P(K)}{P(H)} .
\end{aligned}
$$

By solving this for $P(K)$, we have

$$
P(K) \leq P(H)\left[\frac{1}{C_{\mathrm{O}}\left(\left\{H, H^{*}\right\}\right)}-1\right] .
$$


Now using the inequality (2), we obtain

$$
P(K) \leq P(H)[P(E \mid H)-P(E)]=P(E \wedge H)-P(E) P(H) .
$$

2. As indicated in Figure 1, we define

$$
\begin{aligned}
& a:=P\left(E \wedge H \wedge \neg H^{*}\right), \quad b:=P\left(\neg E \wedge H \wedge \neg H^{*}\right), \\
& a^{*}:=P\left(E \wedge \neg H \wedge H^{*}\right), \quad b^{*}:=P\left(\neg E \wedge \neg H \wedge H^{*}\right) .
\end{aligned}
$$

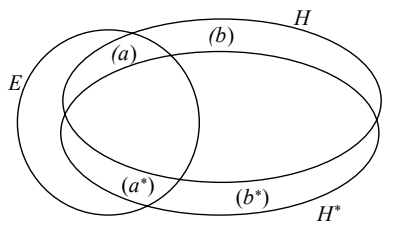

Figure 1: The sets of worlds corresponding to $E, H, H^{*}$ (the probabilities of certain regions are indicated in brackets)

With this notation in place, we can write

$$
\begin{aligned}
P\left(H^{*}\right) & =P(H)+a^{*}+b^{*}-a-b, \\
P\left(H^{*} \mid E\right) & =\frac{P\left(H^{*} \wedge E\right)}{P(E)}=\frac{P(H \wedge E)+a^{*}-a}{P(E)}=P(H \mid E)+\frac{a^{*}-a}{P(E)} .
\end{aligned}
$$

By subtracting the expression for $P\left(H^{*}\right)$ from that for $P\left(H^{*} \mid E\right)$, we thus obtain

$$
\begin{aligned}
D^{*} & =P(H \mid E)+\frac{a^{*}-a}{P(E)}-\left[P(H)+a^{*}+b^{*}-a-b\right] \\
& =P(H \mid E)-a / P(E)-\left[P(H)+b^{*}-a-b\right]+a^{*}(1 / P(E)-1) \\
& >P(H \mid E)-a / P(E)-\left[P(H)+b^{*}\right],
\end{aligned}
$$

where the last inequality is strict because $E$ is not a a tautology. To prove that $D^{*}>0$ it is thus sufficient to show that the last expression is non-negative, i.e. that

$$
P(H \mid E)-a / P(E) \geq P(H)+b^{*}, \text { or } Q:=\frac{P(H \mid E)-a / P(E)}{P(H)+b^{*}} \geq 1 .
$$

To prove that $Q \geq 1$, note first that

$$
Q=\frac{1}{P(E)} \frac{P(E) P(H \mid E)-a}{P(H)+b^{*}}=\frac{1}{P(E)} \frac{P(E \wedge H)-a}{P(H)+b^{*}} .
$$

With $K$ as defined above, we have

$$
a \leq\left(a+a^{*}+b+b^{*}\right)-b^{*}=P(K)-b^{*},
$$

and hence by (3) $a \leq P(E \wedge H)-P(E) P(H)-b^{*}$. So

$$
\begin{aligned}
Q & \geq \frac{1}{P(E)} \frac{P(E \wedge H)-\left(P(E \wedge H)-P(E) P(H)-b^{*}\right)}{P(H)+b^{*}} \\
& =\frac{1}{P(E)} \frac{P(E) P(H)+b^{*}}{P(H)+b^{*}} \geq \frac{1}{P(E)} \frac{P(E) P(H)}{P(H)}=1 \text {. }
\end{aligned}
$$


Proof of Theorem 3. Let $E$ confirm $H$, and put $c:=\frac{1}{1+P(E \wedge H)-P(E) P(H)}$. Consider any consistent formula $H^{*}$ such that $C_{\mathrm{F}}\left(\left\{H, H^{*}\right\}\right) \geq c$. We prove the following inequality analogous to (3):

$$
P(K) \leq P(E \wedge H)-P(E) P(H),
$$

where again $K$ is the formula $\left(H \wedge \neg H^{*}\right) \vee\left(\neg H \wedge H^{*}\right)$. This inequality implies that $E$ confirms $H^{*}$, by an argument analogous to step 2 in the proof of Theorem 2 .

Note that $H^{*}$ cannot be a tautology, since otherwise $C_{\mathrm{F}}\left(\left\{H, H^{*}\right\}\right)=1 / 2$ (by the definition of $C_{\mathrm{F}}$ and the fact that $H$ is not a tautology), violating $C_{\mathrm{F}}\left(\left\{H, H^{*}\right\}\right) \geq c$. So $C_{\mathrm{F}}\left(H, H^{*}\right)$ is given by

$$
\begin{aligned}
C_{\mathrm{F}}\left(\left\{H, H^{*}\right\}\right) & =\frac{1}{2}\left[\frac{P\left(H^{*} \mid H\right)-P\left(H^{*} \mid \neg H\right)}{P\left(H^{*} \mid H\right)+P\left(H^{*} \mid \neg H\right)}+\frac{P\left(H \mid H^{*}\right)-P\left(H \mid \neg H^{*}\right)}{P\left(H \mid H^{*}\right)+P\left(H \mid \neg H^{*}\right)}\right] \\
& \leq \frac{1}{2}\left[\frac{1-P\left(H^{*} \mid \neg H\right)}{1+P\left(H^{*} \mid \neg H\right)}+\frac{1-P\left(H \mid \neg H^{*}\right)}{1+P\left(H \mid \neg H^{*}\right)}\right] .
\end{aligned}
$$

Putting $\alpha:=P\left(H^{*} \mid \neg H\right)$ and $\beta:=P\left(H \mid \neg H^{*}\right)$, we thus have

$$
\begin{aligned}
C_{\mathrm{F}}\left(\left\{H, H^{*}\right\}\right) & \leq \frac{1}{2}\left[\frac{1-\alpha}{1+\alpha}+\frac{1-\beta}{1+\beta}\right]=\frac{1}{2}\left[\frac{(1-\alpha)(1+\beta)+(1+\alpha)(1-\beta)}{(1+\alpha)(1+\beta)}\right] \\
& =\frac{1}{2}\left[\frac{1-\alpha+\beta-\alpha \beta+1+\alpha-\beta-\alpha \beta}{1+\alpha+\beta+\alpha \beta}\right] \\
& =\frac{1}{2}\left[\frac{2-2 \alpha \beta}{1+\alpha+\beta+\alpha \beta}\right]=\frac{1-\alpha \beta}{1+\alpha+\beta+\alpha \beta} \leq \frac{1}{1+\alpha+\beta} .
\end{aligned}
$$

Since $\alpha=\frac{P\left(H^{*} \wedge \neg H\right)}{P(\neg H)} \geq P\left(H^{*} \wedge \neg H\right)$ and $\beta=\frac{P\left(H \wedge \neg H^{*}\right)}{P\left(\neg H^{*}\right)} \geq P\left(H \wedge \neg H^{*}\right)$, we have

$$
\alpha+\beta \geq P\left(H^{*} \wedge \neg H\right)+P\left(H \wedge \neg H^{*}\right)=P(K) .
$$

So, by (5),

$$
C_{\mathrm{F}}\left(\left\{H, H^{*}\right\}\right) \leq \frac{1}{1+P(K)} .
$$

As by assumption $C_{\mathrm{F}}\left(\left\{H, H^{*}\right\}\right) \geq c$, it follows that

$$
\frac{1}{1+P(K)} \geq c=\frac{1}{1+P(E \wedge H)-P(E) P(H)} \text {. }
$$

This implies (4), as desired.

Proof of Theorem 4. For contradiction, assume that $C_{\mathrm{S}}$ satisfies $\left(\mathrm{CT}^{*}\right)$. By assumption, there exist two (distinct) atomic formulae $A$ and $B$. Define $E$ as $A \wedge B$ and $H$ as $A$. Then $E$ confirms $H$. Let $c$ be a coherence threshold as given in $\left(\mathrm{CT}^{*}\right)$. By non-triviality (see footnote 7 ),

$$
c<\sup _{H^{*}} C_{\mathrm{S}}\left(\left\{H, H^{*}\right\}\right)=\sup _{H^{*}} \frac{P\left(H \wedge H^{*}\right)}{P(H) P\left(H^{*}\right)}=\frac{1}{P(H)},
$$

where the last equality holds because $C_{\mathrm{S}}\left(\left\{H, H^{*}\right\}\right)=\frac{P\left(H \wedge H^{*}\right)}{P(H) P\left(H^{*}\right)}$, which is at most $\frac{1}{P(H)}$, and exactly $\frac{1}{P(H)}$ in case $H^{*} \vDash H$ (since then $\left.P\left(H \wedge H^{*}\right)=P\left(H^{*}\right)\right)$. Let $H^{*}$ be $A \wedge \neg B$. By $H^{*} \vDash H$, we have $C_{\mathrm{S}}\left(\left\{H, H^{*}\right\}\right)=\frac{1}{P(H)} \geq c$. Yet $E$ does not confirm $H^{*}$, since $H^{*}$ is inconsistent with $E$. 


\section{References}

Akiba, K. (2000), "Shogenji's Probabilistic Measure of Coherence is Incoherent", Analysis 60: 356-359.

BonJour, L. (1985), The Structure of Empirical Knowledge. Cambridge MA: Harvard University Press.

Bovens, L. and S. Hartmann (2004), Bayesian Epistemology. Oxford: Oxford University Press.

Bovens, L. and S. Hartmann (2003), Solving the Riddle of Coherence, Mind 112(448): 601-633.

Cross, C. (1999), "Coherence and Truth Conducive Justification", Analysis 59: 186-193.

Eells, E. and B. Fitelson (2000), "Measuring Confirmation and Evidence", Journal of Philosophy XCVII(12): 663-672.

Fitelson, B. (2001), "A Bayesian Account of Independent Evidence with Application", Philosophy of Science 68 (Proceedings): 123-140.

Fitelson, B. (2003), "A Probabilistic Theory of Coherence", Analysis 63: 194-199.

Fitelson, B. (2004), "Two technical corrections to my coherence measure", presented at the Bayesian Epistemology Conference, London School of Economics and Political Science, June 2004.

Hoefer, C. and Rosenberg, A. (1994), "Empirical Equivalence, Underdetermination, and Systems of the World", Philosophy of Science 61: 592-607.

Kemeny, J. and P. Oppenheim (1952), "Degrees of Factual Support", Philosophy of Science 19: 307-324.

Klein, P. and T. Warfield (1994), "What Price Coherence?", Analysis 54: 129-132.

Klein, P. and T. Warfield (1996), "No Help for the Coherentist", Analysis 56: $118-121$.

Laudan, L. and Leplin, J. (1991), "Empirical Equivalence and Underdetermination", The Journal of Philosophy 88: 449-472.

Lewis, C. I. (1946), An Analysis of Knowledge and Valuation. La Salle: Open Court.

Merricks, T. (1995), "On Behalf of the Coherentist", Analysis 55: 306-309.

Okasha, S. (1997), "Laudan and Leplin on Empirical Equivalence", The British Journal for the Philosophy of Science 48: 251-256.

Olsson, E. (2002), "What is the Problem of Coherence and Truth?", Journal of Philosophy 94: 246-272.

Olsson, E. (2001), "Why Coherence is Not Truth Conducive", Analysis 61: 236241.

Shogenji, T. (1999), "Is Coherence Truth-Conducive?". Analysis 59: 338-345.

Shogenji, T. (2001), "Reply to Akiba on the Probabilistic Measure of Coherence", Analysis 61: 147-150. 